\title{
Interaction between two localized fluidization cavities in a granular media: experiments and numerical simulation
}

\author{
Jeff Ngoma $^{* 1}$, Stephane Bonelli ${ }^{1}$, Pierre Philippe ${ }^{1}$, J-Y. Delenne ${ }^{2}$, F. Radjai ${ }^{3}$ \\ ${ }^{1}$ National Research Institute of Science and Technology for Environment and Agriculture, \\ OHAX, Aix en Provence (France) \\ ${ }^{2}$ National Institute for Agriculture Research - IATE, Montpellier (France) \\ ${ }^{3}$ Laboratory of Civil and Mechanical Engineering, University of Montpellier 2 (France)
}

In this work, we present experimental and numerical results of the interaction between two localized fluidization cavities in an immersed granular packing. According to the gap between the two locally injected upward fluid flows, each fluidized cavity will evolve independently of the another, or conversely, the two cavities can interact with each other: they merge and eventually behave like a single cavity. Combined optical techniques are used for visualization of particle motion inside the granular media (Refractive index-matching between liquid and beads and planar laser-induced fluorescence), while our two-dimensional simulation is based on coupled Discrete Element and Lattice Boltzmann Methods (DEM-LBM) [1].

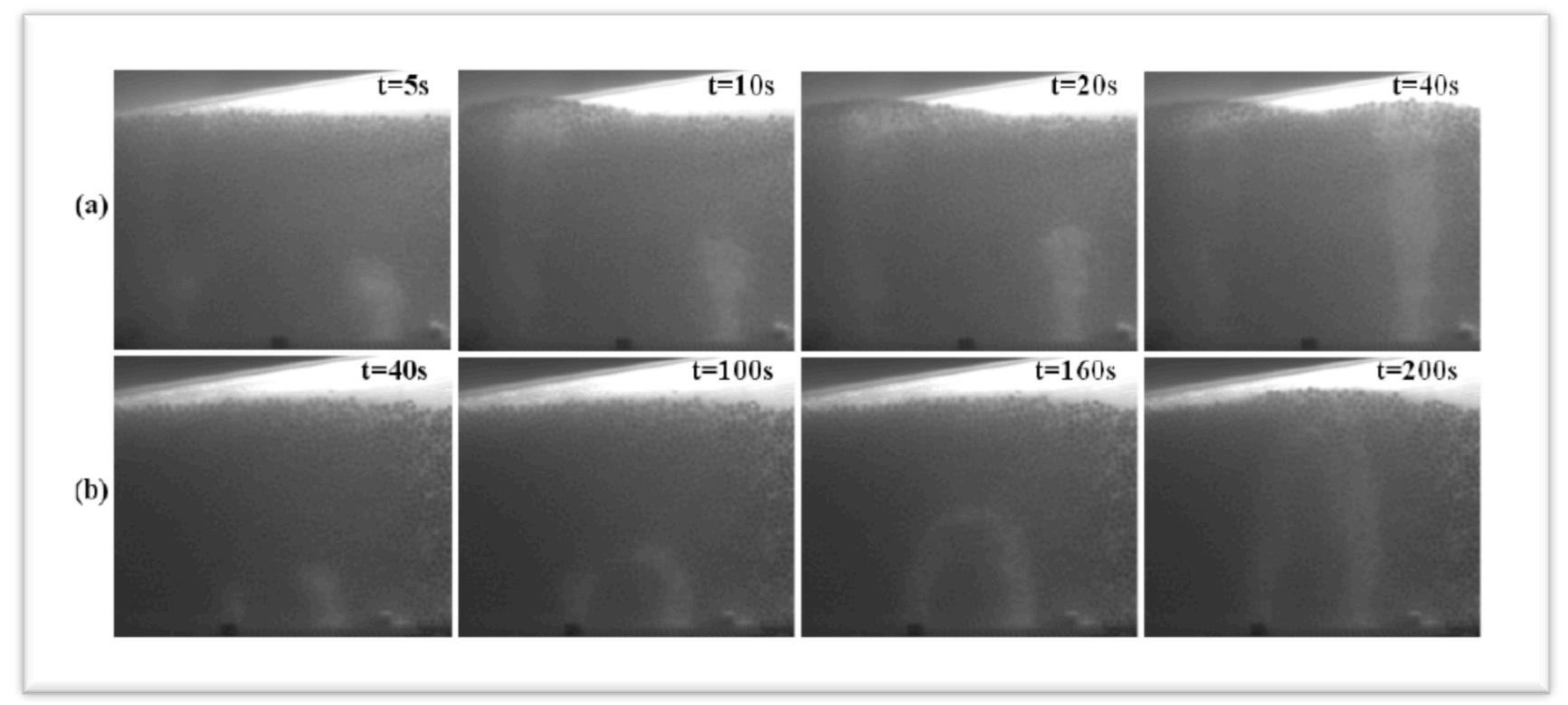

Figure: Successive stages during a sequence of fluidization of a $3 \mathrm{~mm}$ beads layer of initial height $H_{0}=9.2 \mathrm{~cm}$ by to locally holes spaced and with a constant flow rate: (a) large gap; (b) small gap (P. Philippe 2013) [2].

[1] F. Radjai, F. Dubois, Modélisation numérique discrete des matériaux granulaires, Lavoisier, Hermès, Paris, 2010. ISBN 9782746229761.

[2] P. Philippe, M. Badiane, Localized fluidization in granular medium, Physical Review E 87, 042206, 2013. 